\title{
Nurse-performed ultrasound: a new weapon against COVID-19
}

\author{
Jianhua Sun ${ }^{1}$, Qi Li ${ }^{1}$, Xinjuan $\mathrm{Wu}^{2}$, Xiaoting Wang ${ }^{1 *}$ and Dawei Liu ${ }^{1}$
}

Dear Editor,

During the COVID-19 outbreak, nurses were critical to saving patients' lives and improving medical outcomes. Because the disease was highly contagious, protective clothing and gloves were worn in nursing practice. Many procedures, such as peripheral vein puncture and blood collection, were difficult to do. Ultrasound was being performed by doctors to guide hemodynamic monitoring and lung evaluation in ICU [1]. Besides, nurses could use ultrasound to solve difficulties in nursing, such as ultrasound-guided vascular puncture, chest physical therapy, and gastric residual volume (GRV) measurement (Fig. 1). Ultrasound was making nursing easier in critically care. In this letter, we summarize the application of ultrasound in patients of COVID19.

\section{Ultrasound: a useful tool for nursing procedures}

Nurse applied ultrasound to visualize the tip of the catheter and target the vessels in real time and increase the success rate [2]. During the placement of a feeding tube, nurses can identify the esophagus and airway through ultrasound for the first time.
Similarly, ultrasound was useful to the placement of the jejuna nutrition tube [3]. The application of ultrasound helps to avoid risks and optimize care procedures.

\section{Ultrasound: qualitative and quantitative assessments for nursing}

Through ultrasound, nurses can carry out hemodynamic assessment, lung assessment, GRV measurement, thrombosis screening, and so on. Take GRV as an example. Studies reported a good correlation between the gastric antral cross-sectional area and GRV [4]. Ultrasound could dynamically assess GRV and the contraction of the gastric antrum. Ultrasound was performed to assess the patient's condition and implement effective nursing measures.

\section{Ultrasound: bedside decision-making for nurses}

Dyspnea was common in patients with COVID-19. Nurses play an important role in lung care. $90.5 \%$ of acute respiratory failure can be accurately diagnosed within 3 min by BLUE protocol. For patients with lung consolidation, goal-oriented chest physiotherapy could be applied based on ultrasound signs [5]. For patients

\footnotetext{
* Correspondence: icuting@163.com

'Department of Critical Care Medicine, Peking Union Medical College Hospital, Chinese Academy of Medical Sciences, Beijing 100730, China Full list of author information is available at the end of the article
} 


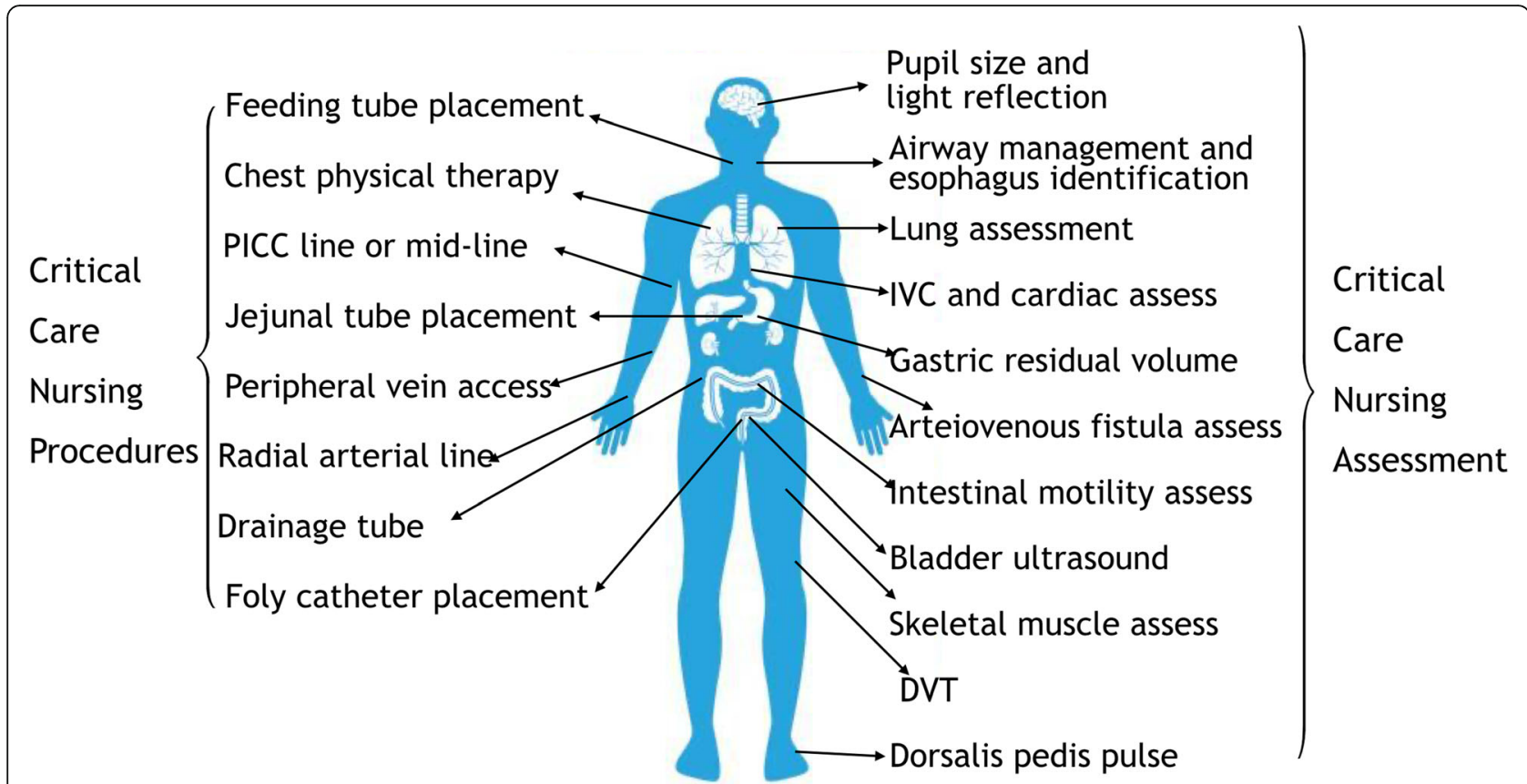

Fig. 1 Ultrasound-guided nursing procedures and assessment in critical care

with pulmonary interstitial syndrome, nurses need to pay more attention to indicators such as edema, inflow and outflow, and CVP.

In this COVID-19 outbreak, the application of ultrasound in patients involves ultrasound-led nursing assessment and ultrasound-guided nursing procedures. Ultrasound was helpful to solve existing problems in nursing practice and guide bedside decision-making. Ultrasound has now become easily available and nurses could benefit from integrating this technology into their clinical practice when taking care of the critically ill.

Acknowledgements

None.

\section{Authors' contributions}

Wang Xiaoting and Liu Dawei conceived and designed the study and interpreted the data. Li Qi made the picture and revised the article. Wu Xinjuan helped interpret the data and drafted the manuscript. Sun Jianhua participated in the study conception and design and drafted the manuscript. All of the authors read and approved the final manuscript.

\section{Funding}

None.

\section{Availability of data and materials}

The datasets used and analyzed during the current study are available from the corresponding author on reasonable request.

Ethics approval and consent to participate None.

\section{Competing interests}

The authors declare that they have no competing interests.

\section{Author details}

${ }^{1}$ Department of Critical Care Medicine, Peking Union Medical College Hospital, Chinese Academy of Medical Sciences, Beijing 100730, China. ${ }^{2}$ Nursing Department, Peking Union Medical College Hospital, Chinese Academy of Medical Sciences, Beijing 100730, China.

Received: 22 May 2020 Accepted: 7 July 2020

Published online: 14 July 2020

\section{References}

1. Tulleken AM, Gelissen H, Lust E, Smits T, van Galen T, Girbes A, Tuinman PR, Elbers P. UltraNurse: teaching point-of-care ultrasound to intensive care nurses. Intensive Care Med. 2019:45(1):1-3.

2. Lamperti M, Bodenham AR, Pittiruti M, Blaivas M, Augoustides JG, Elbarbary M, Pirotte T, Karakitsos D, Ledonne J, Doniger S, et al. International evidence-based recommendations on ultrasound-guided vascular access. Intensive Care Med. 2012:38(7):1105-17.

3. Zhang Q, Sun JH, Liu JT, Wang XT, Liu DW. Placement of a jejunal feeding tube via an ultrasound-guided antral progressive water injection method. Chinese Med J. 2018;131(14):1680-5.

4. Perlas A, Mitsakakis N, Liu L, Cino M, Haldipur N, Davis L, Cubillos J, Chan V. Validation of a mathematical model for ultrasound assessment of gastric volume by gastroscopic examination. Anesth Analg. 2013;116(2):357-63.

5. Wang XT, Ding X, Zhang HM, Chen H, Su LX, Liu DW. Lung ultrasound can be used to predict the potential of prone positioning and assess prognosis in patients with acute respiratory distress syndrome. Crit Care. 2016;20(1): 385.

\section{Publisher's Note}

Springer Nature remains neutral with regard to jurisdictional claims in published maps and institutional affiliations. 